\title{
Narrow Filtered DPSK Implements Order-1 CAPS Optical Line Coding
}

\author{
E. Forestieri, Member, IEEE, and G. Prati, Fellow, IEEE
}

\begin{abstract}
A novel family of optical line codes has been presented elsewhere, here referred to as combined amplitude-phase shift (CAPS) codes. We show here that narrow filtering of a differential phase shift keying signal with bandwidth equal to about $2 / 3$ of the bit rate turns out to closely implement the order-1 CAPS line coding. Performance of the two systems is compared for various types of optical filters.
\end{abstract}

Index Terms-Bandwidth compression, codes, optical fiber communication, optical fiber dispersion, optical modulation, optical propagation in dispersive media.

\section{INTRODUCTION}

$\mathbf{R}$ ECENTLY, modulation formats different from standard on-off keying (OOK) have been considered for transmission in order to increase tolerance to impairments such as chromatic dispersion [causing group velocity dispersion (GVD)], polarization mode dispersion (PMD) and fiber nonlinearities [1], [2]. However, with these modulation formats, the receiver cannot be a standard optically preamplified one, as a Mach-Zehnder interferometer is needed to allow for direct detection of such signal formats. Instead, such a standard receiver can be used in the case of duobinary [3], [4] or derived [5]-[7], coding, the only difference lying in this case in the generation of the coded signals. Whereas duobinary, phase-shaped binary transmission (PSBT) [6] or phased amplitude-shift signaling [7] coding can be achieved through standard Mach-Zehnder modulators, it is not so simple a matter as regards CAPS coding [5]. In [8] (and successively in [9]), it was recognized that narrow filtering a differential phase shift keying (DPSK) signal produces a waveform similar to a duobinary signal without the problems generated by long words, seemingly associated with the imperfect linearity of the amplifiers [10] and the unavoidable asymmetry of the balanced dual-arm modulator [11]. We show here that narrow filtering a DPSK signal produces a waveform closely approximating the order-1 CAPS code signal rather than duobinary.

\section{DPSK AND ORDER-1 CAPS CODE SIGNALS}

In the DPSK, the transmitted optical signal $x_{\mathrm{DPSK}}(t)$ is

$$
x_{\mathrm{DPSK}}(t)=E_{0} \cos \left(\omega_{0} t+\varphi(t)\right)
$$

Manuscript received March 24, 2003; revised September 22, 2003. This work was carried out under Grant by Marconi Communications SpA.

The authors are with the Scuola Superiore Sant' Anna, I-56127 Pisa, Italy, and also with Photonic Networks National Laboratory, CNIT, I-56124 Pisa, Italy (forestieri@sssup.it).

Digital Object Identifier 10.1109/LPT.2003.822231
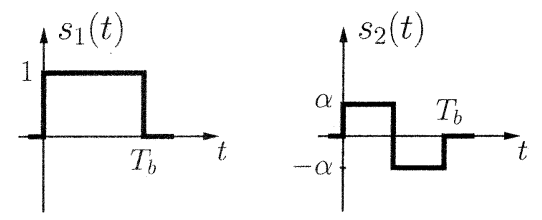

Fig. 1. Elementary signals for order-1 CAPS line code. The factor $\alpha<1$ is a design parameter.

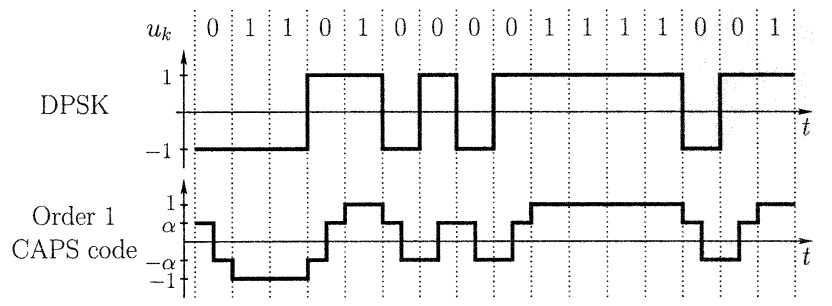

Fig. 2. Example of DPSK and order-1 CAPS code waveforms.

where $\omega_{0}$ is the optical angular frequency carrier, $E_{0}$ is the optical field amplitude and

$$
\varphi(t) \triangleq \sum_{k} \varphi_{k} p\left(t-k T_{b}\right)
$$

$p(t)$ being an ideal nonreturn-to-zero (NRZ) pulse, $T_{b}$ the bit time, and $\varphi_{k}=\varphi_{k-1} \pm\left(u_{k}-1\right) \pi$, where $u_{k} \in\{0,1\}$ is the information symbol.

On the other side, the order-1 CAPS line coding produces a signal which can be written as

$$
x_{\mathrm{CAPS}}(t)=E_{0} \sum_{k} g_{1}\left(\sigma_{k}\right) s_{e}\left(t-k T_{b} ; u_{k}\right) \cos \omega_{0} t
$$

where ${ }^{1}$

$$
s_{e}\left(t ; u_{k}\right)= \begin{cases}s_{1}(t), & \text { if } u_{k}=1 \\ s_{2}(t), & \text { if } u_{k}=0\end{cases}
$$

where $s_{1}(t)$ and $s_{2}(t)$ are the signals depicted in Fig. 1, and $g_{1}\left(\sigma_{k}\right)= \pm 1$ according to the encoder state $\sigma_{k}$ [5].

As an example, the lowpass equivalent signals corresponding to (1) and (3) for the information sequence

$$
\{0,1,1,0,1,0,0,0,0,1,1,1,1,0,0,1\}
$$

are shown in Fig. 2. From Fig. 2, we see that by setting $\alpha=0$, the order-1 code waveform reduces to a duobinary waveform [5], whereas by setting $\alpha=1$, it becomes a DPSK waveform (delayed by $T_{b} / 2$ ). It is also apparent that the two waveforms may become very similar when both are properly filtered, the DPSK by a narrower filter than the other. Indeed, narrow filtering a DPSK signal leaves essentially unaltered its envelope

\footnotetext{
${ }^{1}$ Notice that, for comparison purposes, we are reversing the logic used in [5].
} 

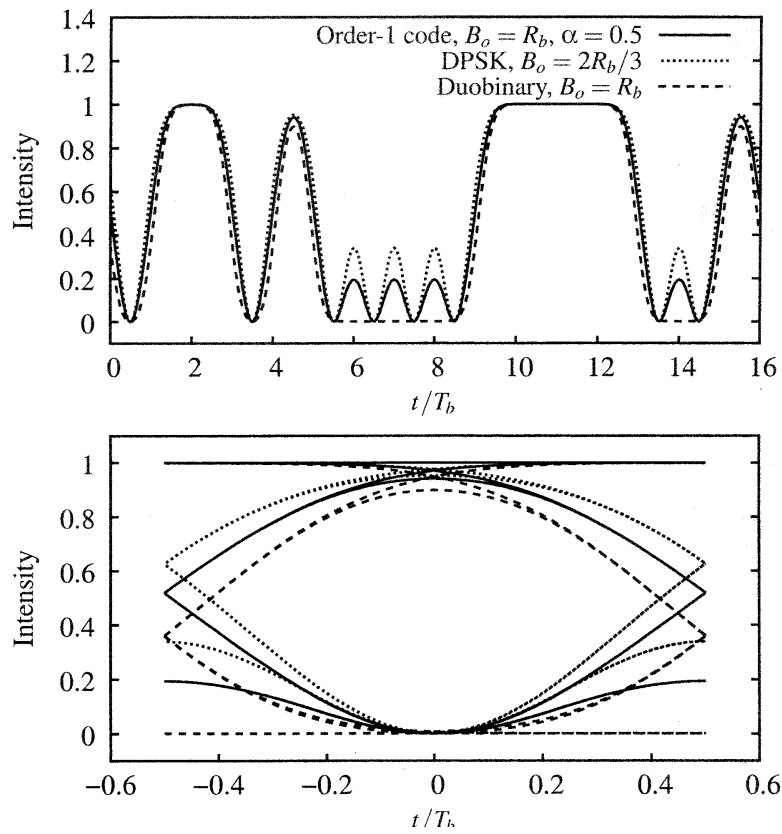

Fig. 3. Intensity and eye pattern of DPSK, order-1 CAPS code, and duobinary waveforms, filtered by a Gaussian-shaped optical filter with 3-dB bandwidth $B_{o}$, corresponding to the $2^{4}$-bit de Bruijn sequence "0 110100001111001 ".

corresponding to a stream of " 1 " (as in this case, no phase shifts take place) and substantially reduces it in correspondence of a stream of " 0 " (as in this case, a phase shift takes place at each bit time).

In Fig. 3, the intensity and corresponding eye pattern of a DPSK, order-1 CAPS code, and duobinary signals filtered by an optical Gaussian-shaped filter with a 3-dB bandwidth equal to $B_{o}=2 R_{b} / 3$ for DPSK and $B_{o}=R_{b}$ for the other cases, are shown ( $R_{b}=1 / T_{b}$ is the bit rate). As can be seen, the first two waveforms are very similar, confirming that the order-1 CAPS code can be implemented by narrow filtering a DPSK signal. In fact, in Fig. 3, the optimum value of $\alpha=0.5$ was used for the order-1 CAPS code [5], but had we used a slightly larger value for $\alpha$, the two waveforms would have been practically indistinguishable from each other. Also notice that the waveforms reported in Fig. 3 correspond to an infinite bandwidth postdetection filter. In an optically preamplified system, the postdetection filter is needed to further limit the noise variance when the predetection optical filter is not too narrow, otherwise its presence could even be detrimental as it would cause an eye closure not counterbalanced by a noise variance reduction. ${ }^{2}$ In standard OOK, such an optical filter cannot be made too narrow and its bandwidth (together with the postdetection filter bandwidth) is the result of a compromise between intersymbol interference and noise rejection. In the DPSK case, the receive optical filter must be much narrower to obtain the desired signal shaping and thus it is not surprising that the postdetection filter is not needed.

\section{PERformance EVAluation}

The optical filter turns out to be quite important in determining the performance. We found that this filter should have a

${ }^{2}$ When amplified spontaneous emission dominates thermal and shot noise, i.e., for a high-gain amplifier.
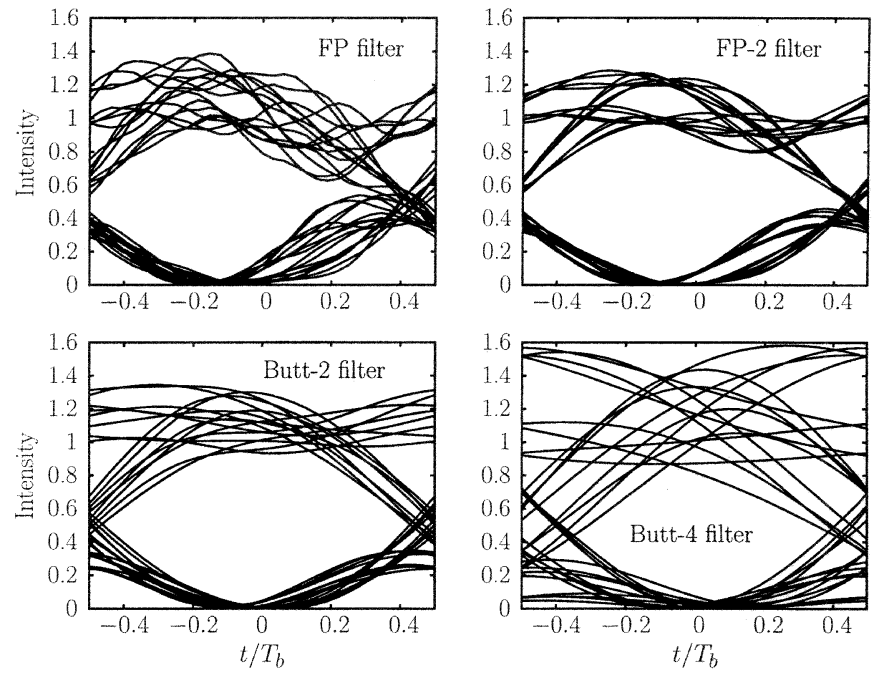

Fig. 4. Eye patterns of a square law detected $10 \mathrm{~Gb} / \mathrm{s}$ DPSK signal narrow filtered with various optical filters with $3-\mathrm{dB}$ bandwidth $B_{o}=2 R_{b} / 3$ after $100 \mathrm{~km}$ of standard fiber and no postdetection filtering.

not too sharp or smooth passband skirt and its phase response should be as much linear as possible. We have compared several types of filters, ranging from high-finesse Fabry-Perot (FP), cascade of identical FP filters (FP-2), second- and fourth-order maximally flat (or Butterworth) to uniform fiber Bragg grating (UFBG) filter. The first three filters are described in [12]. In Fig. 4, the eye patterns corresponding to the first four filters are shown after $100 \mathrm{~km}$ of standard fiber with a chromatic dispersion of $17 \mathrm{ps} / \mathrm{nm} / \mathrm{km}$ at $R_{b}=10 \mathrm{~Gb} / \mathrm{s}$. As can be seen, the FP and Butt-4 filters produce worse results than the FP-2 and Butt-2 filters, because they have too smooth (FP filter) or too sharp (Butt-4 filter) passband skirts. As regards the UFBG filter, it is characterized by its induced change in the core-mode effective index $\overline{\delta n}_{\text {eff }}(z)$, coupling coefficient $\kappa$, and length $L$ [13], [14]. This kind of filter has sidelobes whose amplitude depends on $\kappa L$ as the amplitude response is roughly proportional to the Fourier transform of $\overline{\delta n}_{\mathrm{eff}}(z)$ [15], which is of rectangular shape for uniform gratings. Smaller values of $\kappa L$ result in smoother passband skirts, higher sidelobe suppression, and increased phase response linearity. We found that an UFBG filter produces acceptable results only for $\kappa L \leq 1$, i.e., for a sidelobe suppression ratio of more than about $11 \overline{\mathrm{dB}}$.

We have considered the effect of postdetection filtering by evaluating the bit error rate $^{3}$ (BER) with the approach given in [16]. In Fig. 5, the BER is reported as a function of the postdetection electrical filter bandwidth, taken as a fifth-order Bessel filter, for a fixed value of the optical signal-to-noise ratio (OSNR) at the output of the optical high-gain preamplifier. It can be seen that in the FP and UFBG cases, the behavior is as expected when the postdetection filter bandwidth $B_{e}$ increases, whereas this is not the case for the other optical filters where the BER steadily decreases. This is due to the fact that when increasing $B_{e}$ the upper rails are spread more apart for the FP and UFBG filters causing a signal dependent noise variance increase which overwhelms the improved eye opening. The best optical filter turns out to be the Gaussian-shaped one, followed

${ }^{3}$ We use the current terminology instead of the more correct "bit error probability." 


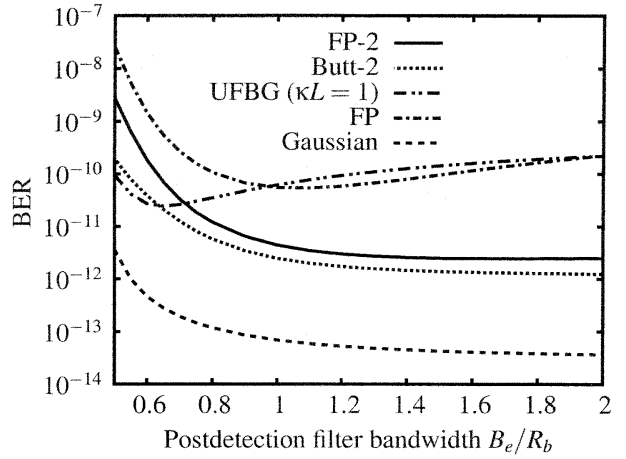

Fig. 5. BER for a square law detected $10 \mathrm{~Gb} / \mathrm{s}$ DPSK signal (2 $2^{11}$-bit de Bruijn sequence [16]) narrow filtered by various optical filters with 3-dB bandwidth $B_{o}=2 R_{b} / 3$, after $100 \mathrm{~km}$ of standard fiber. Postdetection filter is fifth-order Bessel.

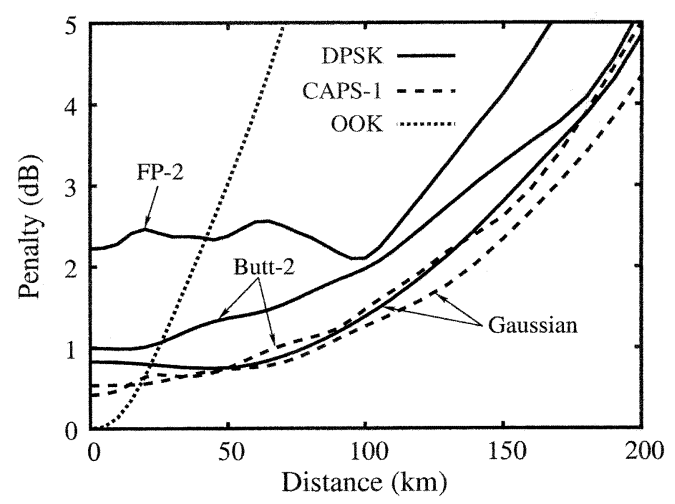

Fig. 6. OSNR penalty at BER $=10^{-12}$ as a function of fiber length, for a square law detected $10 \mathrm{~Gb} / \mathrm{s}$ DPSK and CAPS-1 code signal ( $2^{11}$-bit de Bruijn sequence [16]) narrow filtered by various optical filters with 3-dB bandwidth $B_{o}=2 R_{b} / 3$ and $B_{o}=R_{b}$, respectively. No postdetection filtering is performed. Penalty for reference OOK system is superimposed.

by the Butt-2 filter which is practically equivalent to the cascade of two FP filters for a fiber length of $100 \mathrm{~km}$.

In Fig. 6, the OSNR penalty at BER $=10^{-12}$ and $R_{b}=10 \mathrm{~Gb} / \mathrm{s}$ is reported as a function of the fiber length. The reference system is a standard OOK optical system in a back-to-back configuration with a Gaussian optical filter and fifth-order Bessel postdetection filter, whose bandwidths of $2 R_{b}$ and $0.6 R_{b}$, respectively, are jointly optimized for minimum OSNR at the given BER. The optical filter has bandwidth $B_{o}=2 R_{b} / 3$ for DPSK, and $B_{o}=R_{b}$ for the order-1 CAPS code, whereas no postdetection filtering is performed in both cases. Indeed, we verified that the postdetection filter is also not necessary in the case of CAPS coding even if a slightly larger optical filter than for the DPSK case is used. Notice that for CAPS coding the tight filtering is performed by the optical filter, rather than through baseband filtering of the modulating signal as done in [5], as this allows for improved sensitivity. We also evaluated the penalty for CAPS coding with Butt-2 and FP-2 optical filters, the latter not reported in Fig. 6 to avoid clutter. It turned out that CAPS coding is less sensitive to the optical filter as the penalties obtained with the Butt-2 and FP-2 filters are within $0.2 \mathrm{~dB}$ and $0.7 \mathrm{~dB}$, respectively, from that obtained with Gaussian filtering. Fig. 6 shows that, when properly filtered, the DPSK signal performance is very close to that of the CAPS code signal. As a matter of fact, the optical filter type plays an important role and penalties in excess of $1 \mathrm{~dB}$ should be expected when its shape is too much different from the optimal Gaussian shape.

\section{CONCLUSION}

CAPS coding is very effective in combating chromatic dispersion [5] but the required combined amplitude and phase modulated signal is not easy to generate. Reinterpreting previous findings [8], [9], we show that this complex modulated signal can be approximated for order-1 CAPS coding by narrow filtering a DPSK signal, which is much easier to generate. However, to obtain the predicted performance, the optical filter should have a Gaussian shape and a linear phase response. Such narrowband Gaussian-shaped optical filters could be obtained by Gaussian apodized FBG filters [14].

\section{REFERENCES}

[1] R. A. Griffin and A. C. Carter, "Optical differential quadrature phaseshift key (oDPQSK) for high capacity optical transmission," in Proc. OFC 2002, 2002, Paper WX6, pp. 367-368.

[2] W. Christoph, L. Jochen, and R. Werner, "RZ-DQPSK format with high spectral efficiency and high robustness toward fiber nonlinearities," in Proc. ECOC 2002, 2002, Paper 9.6.6.

[3] A. J. Price, L. Pierre, R. Uhel, and V. Havard, "210-km repeaterless $10-\mathrm{Gb} / \mathrm{s}$ transmission experiment through nondispersion-shifted fiber using partial response scheme," IEEE Photon. Technol. Lett., vol. 7, pp. 1219-1221, Oct. 1995.

[4] S. Kuwano, K. Yonenaga, and K. Iwashita, " $10 \mathrm{Gbit} / \mathrm{s}$ repeaterless transmission experiment of optical duobinary modulated signal," Electron. Lett., vol. 31, no. 16, pp. 1359-1361, 1995.

[5] E. Forestieri and G. Prati, "Novel optical line codes tolerant to fiber chromatic dispersion," J. Lightwave Technol., vol. 19, pp. 1675-1684, Nov. 2001.

[6] D. Penninckx, M. Chbat, L. Pierre, and J.-P. Thiery, "The phase-shaped binary transmission (PSBT): A new technique to transmit far beyond the chromatic dispersion limit," IEEE Photon. Technol. Lett., vol. 9, pp. 259-261, Feb. 1997.

[7] J. B. Stark, J. E. Mazo, and R. Laroia, "Line coding for dispersion tolerance and spectral efficiency: Duobinary and beyond," in Proc. OFC'99, vol. WM46-1, 1999, pp. 331-333.

[8] A. Røyset and D. R. Hjelme, "Novel dispersion tolerant optical duobinary transmitter using phase modulator and Bragg grating filter," in Proc. ECOC'98, vol. 1, 1998, pp. 225-226.

[9] D. Penninckx, H. Bissessur, P. Brindel, E. Gohin, and F. Bakhti, "Optical differential phase shift keying (DPSK) direct detection considered as a duobinary signal," in Proc. ECOC'01, vol. 3, 2001, pp. 456-457.

[10] T. Frank, P. B. Hansen, T. N. Nielsen, and L. Eskildsen, "Novel duobinary transmitter," in Proc. ECOC'97, vol. 1, 1997, pp. 67-70.

[11] A. Røyset and D. R. Hjelme, "Symmetry requirements for 10-Gb/s optical duobinary transmitters," IEEE Photon. Technol. Lett., vol. 10, pp. 273-275, Feb. 1998.

[12] A. A. M. Saleh and J. Stone, "Two-stage Fabry-Perot filters as demultiplexers in optical FDMA LANs," J. Lightwave Technol., vol. 7, pp. 323-330, Feb. 1989.

[13] A. Othonos and K. Kalli, Fiber Bragg Gratings. Norwell, MA: Artech House, 1999.

[14] T. Erdogan, "Fiber grating spectra," J. Lightwave Technol., vol. 18, pp. 1277-1294, Aug. 1997.

[15] K. O. Hill and G. Meltz, "Fiber Bragg grating technology fundamentals and overview," J. Lightwave Technol., vol. 18, pp. 1263-1276, Aug. 1997.

[16] E. Forestieri, "Evaluating the error probability in lightwave systems with chromatic dispersion, arbitrary pulse shape and pre- and postdetection filtering," J. Lightwave Technol., vol. 18, pp. 1493-1503, Nov. 2000. 